\title{
Study of Second-Order Continuities of a Composite Surface
}

\author{
Yukun Liu', Yong Yue ${ }^{2}$ \\ ${ }^{1}$ Department of Computer, School of Information Science and Engineering, Hebei University of Science and Technology, \\ Shijiazhuang, China \\ ${ }^{2}$ Department of Computer Science and Software Engineering, Xi'an Jiaotong-Liverpool University, Suzhou, China \\ Email:1yklucky@sina.com, lyklucky@hebust.edu.cn,yong.yue@xjtlu.edu.cn
}

How to cite this paper: Liu, Y.K. and Yue, Y. (2020) Study of Second-Order Continuities of a Composite Surface. Advances in Pure Mathematics, 10, 21-38. https://doi.org/10.4236/apm.2020.101003

Received: December 23, 2019

Accepted: January 13, 2020

Published: January 16, 2020

Copyright (C) 2020 by author(s) and Scientific Research Publishing Inc. This work is licensed under the Creative Commons Attribution International License (CC BY 4.0).

http://creativecommons.org/licenses/by/4.0/

\section{(c) (i) Open Access}

\begin{abstract}
There are two classes of continuities, parametric continuities and geometric continuities, which are used to illuminate the smoothness of a composite surface in surface construction and reconstruction in computer graphics (CG) and computer aided design (CAD). A parametric continuity is more stiff than its corresponding geometric continuity of the same order. This paper uncovers the geometric properties of parametric and geometric continuities less than and equal to second order and presents the proofs for the corresponding propositions. These propositions can be applied to the existent or promising schemes of surface construction or reconstruction, which can provide a convincing theory for researchers to establish their schemes in surface construction. Three examples are used in this paper to show the applications of these propositions.
\end{abstract}

\section{Keywords}

Parametric Continuities, Geometric Continuities, Composite Surface, Bending Rate, Normal Section Curvature

\section{Introduction}

It is known that there are a lot of applications of surface construction or reconstruction in computer graphics (CG), computer aided design (CAD), computer aided geometric design (CAGD), and their succeeding applications, such as visualization in scientific computing (VISC), medical imagining technology (MIT), medical image reconstruction (MIR), manufacturing of ships, aircrafts and cars, digital special effect in film and television animation production, etc. [1] [2]. 
The process of surface construction or reconstruction usually starts from sampling discrete points (vertices) on a target surface that is studied by researchers. The results of surface construction and reconstruction in computer are often composite surfaces which are patches glued together. Researchers create their schemes to construct or reconstruct a composite surface via computer technology to approximate the target surface. Then the constructed or reconstructed surfaces can be applied to education, researches, and management in medicine, biology, geography, mechanics, electrical engineering, science, business, entertainment, and others.

There are two classes of continuities characterized by many researchers to examine how smooth a composite surface is, these being called parametric continuities and geometric continuities [3] [4] [5] [6] [7]. Following the studies of the predecessors [3] [4] [5] [6] [7], this paper provides a further description on the continuities of $r^{\text {th }}$ order, $r \leq 2$, and presents different mathematical features of these continuities.

The required proofs are given along with the discussion, which can verify the study in this paper. The features presented in this paper can be directly applied to examining how smooth a surface can be constructed or reconstructed by an existent or promising scheme. It can save the time and effort for researchers to look for a convincing theory of surface continuities to make their new methods established.

Related studies are given in Section 2. A system of surface construction has to be defined first in the context of algebraic topology and differential geometry in Section 3. After uni-parametric representations of continuities are given in Section 4, bi-parametric formulae of geometric continuities are further presented in Section 5. In Section 6, properties of first-order continuities are discussed. In Section 7, properties of second-order continuities are further extended. In Section 8 , three examples are used to show how to apply the theory of this paper. Section 9 is results and conclusions.

\section{Related Studies}

In $\mathrm{CG}, \mathrm{CAD}, \mathrm{CAGD}$ and other applications of surface construction and reconstruction, researchers work hard to construct composite surfaces as smooth as needed in their applications [3] [4] [5] [6] [7]. Some researchers have noticed that parametric continuities were usually too stiff to meet for a scheme of surface construction. For this reason, Barsky and DeRose [3] [4] devised the Beta-spline surfaces that meet the geometric continuities rather than parametric continuities. According to the studies [3] [4] [5] [6] [7], parametric continuities can shrink the set of parameterizations by excluding ones that can generate geometrically smooth curves or surfaces. The reason for this is that the condition of parametric continuity of a given order is stricter than one of the geometric continuity of the same order, which will be discussed in this paper.

Researchers in applications of surface construction and reconstruction usually 
presented the schemes that could be used to construct a composite surface with the continuity of a given order without a mathematical proof to verify how smooth it is. To our knowledge, no others have done the further study on the geometric properties of these two classes of continuities of a composite surface and the related proofs.

On the other hand, the existent theory of algebraic topology [8] has established the mathematics foundation for the study of this paper, which can be used to present the definition of a system of surface construction in a mathematical way, which will be introduced in Section 3. In this system, our further discussion will progress to the geometric properties of these two classes of continuities. To support the further discussion in this paper, the theories of modern geometry [9] and differential geometry [10] [11] must be introduced. In this way, the study of this paper can bridge the gap between the surface construction and the related mathematics theories.

With the above purpose and theories, this paper uncovers the geometric properties of these two classes of continuities of less than and equal to second order and presents the proofs for the corresponding propositions. These propositions can be applied to the existent or promising schemes of surface construction or reconstruction, which can provide a convincing theory for researchers to establish their schemes in surface construction and reconstruction.

\section{System of Surface Construction}

In order to follow the researches of predecessors mentioned above and save the extra effort in the existent proofs, we put our discussion in the context of the related theory that has been established. A system of surface construction or reconstruction is defined in algebraic topology firstly here. From the point of view of constructing a surface from a network of sampled vertices with the computer technology, surface reconstruction can be included in surface construction. Thus, we will substitute surface construction or reconstruction with surface construction in the rest of this paper.

This paper will develop its deduction on the basis of the existent theory of algebraic topology, especially on the treatise of Massey [8]. Therefore, the definitions here will start from this basis. It is also required to mention that this paper will focus on surfaces that are orientable and locally connected since these types of surfaces are ones usually studied in CG, CAD, CAGD, and their related applications.

Definition 1. A segment $f$ in a surface $S$ is a continuous map of some closed interval into $S$. That is, $f: I \rightarrow S$. Since it is continuous, the closed interval can be set $I=[0,1]$. Two points, $\{a, b\}$, the images of the end points of the interval I denote the end points of the segment, and the segment $f$ is called to join its end points. The segment $f$ is orientable so that $a$, the point from which the segment starts, is called initial point and $b$, the point which the segment reaches, is called terminal point. 
Definition 2. A surface $S$ is called segment-wise connected if any two points of $S$ can be joined by a segment. A segment-wise connected surface is connected. The segment components of $S$ are the maximal segment-wise connected subsets of $S$. A surface is locally segment-wise connected if each point has a basic family of segment-wise connected neighborhoods. A surface that is connected and locally segment-wise connected is segment-wise connected.

Definition 3. Let $S$ be a surface. $S$ is also a topological space. Let $M$ be a system of surface construction. $M$ is a pair $\{\hat{S}, \varphi\}$, where $\hat{S}$ is a constructed surface and $\varphi: \hat{S} \rightarrow S$ a continuous map such that the following condition holds. Each point $x \in S$ has a segment-wise-connected open neighborhood $K$ such that each segment component of $\varphi^{-1}(K)$ is mapped topologically onto $K$ by $\varphi . \varphi^{-1}(K)$ is nonempty. The map $\varphi$ is also called a projection. Any open neighborhood $K$ that meets the above condition is called a prime neighborhood.

Definition 3 is analogous to the definition of the covering space in algebraic topology [8]. The purpose of doing so is to channel surface construction of CG, $\mathrm{CAD}$, and CAGD into algebraic topology of which the established theory can be applied directly to this paper. It is also natural and proper because a surface is one class of topological spaces and a surface constructed by a method in CG, CAD, and CAGD can be corresponding to a covering space of a target surface that is studied in CG, CAD, and CAGD. That is, the covering space in algebraic topology is transferred into the system of surface construction in CG, CAD, and CAGD here. As a constructed surface is a composite surface, they are interchangeable in this paper.

\section{Parametric Continuities and Geometric Continuities}

In order to explore the geometric meanings and properties of smooth conditions between adjacent patches of a composite surface, it is necessary to define two different types of continuities, parametric continuities and geometric continuities, which have been presented for years in CG, CAD, and CAGD [3] [4] [5] [6] [7], and are still used recently [12] [13]. Here, they are defined in the context of the system of surface construction.

The local continuity of a patch is obvious if a set of algebraic formulae with definite continuities are used to express the patch. In CG, CAD and CAGD, researchers usually discuss continuities on the boundaries between pairs of patches when they present their schemes of surface construction. The focus of this paper is put primarily on the boundaries between pairs of patches that are sewed together to form a composite surface.

Let us move onto the definitions of parametric and geometric continuities.

There exists a system of surface construction $\{\hat{S}, \varphi\}$ (defined in Definition 3). $\varphi$ consists of a set of polynomial formulae that interpolate and approximate a target surface $S$ to connect the network of vertices $N$, which are sampled on the target surface $S$, and to construct each patch $c_{i} \subset \hat{S} . \hat{S}$ is the constructed surface of the system of surface construction. 
There exist a pair of adjoining patches $\left\{c_{i}, c_{j}\right\}, c_{i} \subset \hat{S}$ and $c_{j} \subset \hat{S}$. In these two patches, there exist two uni-parametric curve segments, $l_{c i}(u) \subset c_{i}$ and $l_{c j}(w) \subset c_{j}$, where the parameters $u \in\left[u_{0}, u_{1}\right]$ and $w \in\left[w_{0}, w_{1}\right]$, respectively. These two segments satisfy Definition 1 and meet at a common point $p=l_{c i}\left(u_{1}\right)=l_{c j}\left(w_{0}\right)$.

Definition 4. We say that the above two segments, $l_{c i}(u)$ and $l_{c j}(w)$ meet with the $n^{\text {th }}$-order parametric continuities $\left(C^{n}\right)$ if the following conditions are satisfied:

$$
\left.\frac{\mathrm{d}^{k}}{\mathrm{~d} u^{k}} l_{c i}(u)\right|_{u_{1}^{-}}=\left.\frac{\mathrm{d}^{k}}{\mathrm{~d} w^{k}} l_{c j}(w)\right|_{w_{0}^{+}}
$$

where $u_{1}=w_{0}$ and $k=0, \cdots, n$. Especially, the $C^{0}$ condition is

$$
l_{c i}\left(u_{1}\right)=l_{c j}\left(w_{0}\right) \text {. }
$$

Definition 5 We say that the above two curve segments, $l_{c i}$ and $l_{c j}$, meet with the $n^{\text {th }}$-order geometric continuity $\left(G^{n}\right)$ if there exists an algebraic curve $l_{a}$ which meets both curves $l_{c i}$ and $l_{c j}$ at $p$ with contact of order $n$ in the sense that the first $n$ terms in the Taylor series expansions about the point $p$ of the two given segments, $l_{c i}$ and $l_{c j}$, and the algebraic curve $l_{a}$ all agree at $p$.

If these two segments, $l_{c i}$ and $l_{c j}$, join with the parametric continuities with respect to the natural arc length parameterization and with the assistance of the chain rule, $l_{c i}$ and $l_{c j}$ meet the conditions of geometric continuities. That is, the condition of $G^{0}$ is written as,

$$
l_{c i}\left(u_{1}^{-}\right)=l_{c j}\left(w\left(u_{1}^{-}\right)\right)=I_{c j}\left(w_{0}^{+}\right) .
$$

The condition of $G^{1}$ is written as

$$
\left.\frac{\mathrm{d}}{\mathrm{d} u} l_{c i}(u)\right|_{u_{1}^{-}}=\left(\left.\frac{\mathrm{d}}{\mathrm{d} u} w(u)\right|_{u_{1}^{-}}\right)\left(\left.\frac{\mathrm{d}}{\mathrm{d} w} l_{c j}(w)\right|_{w_{0}^{+}}\right)
$$

The condition of $G^{2}$ is written as

$\left.\frac{\mathrm{d}^{2}}{\mathrm{~d} u^{2}} l_{c i}(u)\right|_{u_{1}^{-}}=\left(\left.\frac{\mathrm{d}}{\mathrm{d} u} w(u)\right|_{u_{1}^{-}}\right)^{2}\left(\left.\frac{\mathrm{d}^{2}}{\mathrm{~d} w^{2}} l_{c j}(w)\right|_{w_{0}^{+}}\right)+\left(\left.\frac{\mathrm{d}^{2}}{\mathrm{~d} u^{2}} w(u)\right|_{u_{1}^{-}}\right)\left(\left.\frac{\mathrm{d}}{\mathrm{d} w} l_{c j}(w)\right|_{w_{0}^{+}}\right)$

In CG, $C A D$, and CAGD, parametric continuities $C^{k}$ are usually too stiff for researchers to find a set of parametric expressions that satisfy them in practical situations if $k>1$. Therefore, geometric continuities $G^{k}$ are applied to some schemes of surface constructions [3] [4] [5] [6] [7] [12].

Since patches of a composite surface are usually represented by bi-parametric algebraic expressions in CG, CAGD, and CAD, it is convenient to represent the geometric continuities with bi-parametric formulae. Bi-parametric formulae of geometric continuities will be presented in the next section.

\section{Bi-Parametric Formulae of Geometric Continuities}

Give two bi-parametric patches, $\left\{c_{i}, c_{j}\right\}, \quad c_{i}(u, v) \subset \hat{S}$ and $c_{j}(w, t) \subset \hat{S}$, 
where $\hat{S}$ is a constructed surface. $c_{i}(u, v)$ and $c_{j}(w, t)$ are expressed by radius vectors in differential geometry. These two patches meet at a point, $p=c_{i}(\widehat{u}, \widehat{v})=c_{j}(\widehat{w}, \widehat{t})$. According to Definition 5 and the study of Hoschek and Lasser [7], the condition of zeroth-order geometric continuity $G^{0}$ is as follows,

$$
c_{i}(\widehat{u}, \widehat{v})=c_{j}(\widehat{w}, \widehat{t})=c_{j}(w(\widehat{u}, \widehat{v}), t(\widehat{u}, \widehat{v})),
$$

where $\widehat{w}=w(\hat{u}, \widehat{v})$, and $\hat{t}=t(\hat{u}, \widehat{v})$.

The condition of first-order geometric continuity $G^{1}$ is written as follows,

$$
\dot{c}_{i}(\widehat{u}, \widehat{v})=T_{11} \dot{c}_{j}(\widehat{w}, \widehat{t}),
$$

where $\dot{c}_{i}(\hat{u}, \widehat{v})=\left.\left[\begin{array}{l}\frac{\partial}{\partial u} c_{i} \\ \frac{\partial}{\partial v} c_{i}\end{array}\right]\right|_{(\hat{u}, \hat{v})}, \quad \dot{c}_{j}(\widehat{w}, \widehat{t})=\left.\left[\begin{array}{l}\frac{\partial}{\partial w} c_{j} \\ \frac{\partial}{\partial t} c_{j}\end{array}\right]\right|_{(\hat{w}, \hat{t})}$ and $T_{11}=\left.\left[\begin{array}{ll}\frac{\partial w}{\partial u} & \frac{\partial t}{\partial u} \\ \frac{\partial w}{\partial v} & \frac{\partial t}{\partial v}\end{array}\right]\right|_{(\hat{u}, \hat{v})}$.

The condition of second-order geometric continuity $G^{2}$ is written as follows,

$$
\begin{aligned}
& \ddot{c}_{i}(\widehat{u}, \widehat{v})=T_{22} \ddot{c}_{j}(\widehat{w}, \widehat{t})+T_{12} \dot{c}_{j}(\widehat{w}, \widehat{t}), \\
& \text { where } \ddot{c}_{i}(\hat{u}, \widehat{v})=\left[\left.\begin{array}{c}
\frac{\partial^{2}}{\partial u^{2}} c_{i} \\
\frac{\partial^{2}}{\partial u \partial v} c_{i} \\
\frac{\partial^{2}}{\partial v^{2}} c_{i}
\end{array}\right|_{(\hat{u}, \hat{v})}, \ddot{c}_{j}(\widehat{w}, \widehat{t})=\left.\left[\begin{array}{c}
\frac{\partial^{2}}{\partial w^{2}} c_{j} \\
\frac{\partial^{2}}{\partial w \partial t} c_{j} \\
\frac{\partial^{2}}{\partial t^{2}} c_{j}
\end{array}\right]\right|_{(\hat{w}, \hat{t})}\right. \text {, } \\
& T_{22}=\left[\left.\begin{array}{ccc}
\left(\frac{\partial w}{\partial u}\right)^{2} & 2\left(\frac{\partial w}{\partial u}\right)\left(\frac{\partial t}{\partial u}\right) & \left(\frac{\partial t}{\partial u}\right)^{2} \\
\left(\frac{\partial w}{\partial u}\right)\left(\frac{\partial w}{\partial v}\right) & \left(\frac{\partial w}{\partial u}\right)\left(\frac{\partial t}{\partial v}\right)+\left(\frac{\partial w}{\partial v}\right)\left(\frac{\partial t}{\partial u}\right) & \left(\frac{\partial t}{\partial u}\right)\left(\frac{\partial t}{\partial v}\right) \\
\left(\frac{\partial w}{\partial v}\right)^{2} & 2\left(\frac{\partial w}{\partial v}\right)\left(\frac{\partial t}{\partial v}\right) & \left(\frac{\partial t}{\partial v}\right)^{2}
\end{array}\right|_{(\hat{u}, \bar{v})}\right. \\
& \text { and } T_{12}=\left[\left.\begin{array}{cc}
\frac{\partial^{2} w}{\partial u^{2}} & \frac{\partial^{2} t}{\partial u^{2}} \\
\frac{\partial^{2} w}{\partial u \partial v} & \frac{\partial^{2} t}{\partial u \partial v} \\
\frac{\partial^{2} w}{\partial v^{2}} & \frac{\partial^{2} t}{\partial v^{2}}
\end{array}\right|_{(\hat{u}, \bar{v})}\right.
\end{aligned}
$$

Before discussing the properties of continuities, let us see how to represent the first and second derivatives in Equations ((3), (4), (6) and (7)) in the discrete way in CG, CAGD, and CAD in order to see which points in the neighborhood of an assessed point should be involved in computing with computer technology. In practice, these points are influenced by a concrete scheme of surface construction [3] [4] [5] [6] [7].

It is necessary to notice that the first derivatives in Equations ((3), (4), (6) and 
(7)) can be represented approximately with the ratio of the difference of corresponding function values of two points to the parametric variation in CG, CAGD, and CAD. In this way, the first column of points on both sides in the neighborhood of the assessed point $p$ is involved in computing the first derivatives, shown in Figure 1. They are $p_{1}$ and $p_{2}$.

In an analogous way, the second derivatives in Equations (4) and (7) can be represented approximately with the ratio of the difference of the corresponding first derivatives to the parametric variation. Thus, the first and second columns of points on both sides in the neighborhood of the assessed point are involved in computing the second derivatives. They are $p_{1}, p_{2}, p_{3}$ and $p_{4}$. Figure 1 shows the first and second columns of points on both sides in the neighborhood of an assessed point $p$.

We will explore the properties of first order continuities in Section 6 and the properties of second order continuities in Section 7, respectively.

\section{Properties of First-Order Continuities}

Remark 1. Let us see Equations (1) and (3) in Section 3, and compare the conditions of first-order parametric continuity $C^{1}$ and first-order geometric continuity $G^{1}$ of two uni-parametric curves. Since the difference between them is the derivative argument, $\left.\frac{\mathrm{d}}{\mathrm{d} u} w(u)\right|_{u_{1}^{-}}$, this argument expands the identity of derivative vectors of the same orientation and scale into the equivalence of derivative vectors of only the same orientation in three-dimensional space $R^{3}$. The scale difference can be complemented by an extra transformation or mapping, or it can be held for two adjoining patches to have distinguishing appearances when crossing their boundary.

The idea of Remark 1 can be further expanded into bi-parametric curve segments in two adjoining patches of a surface with two more conditions so that the patches are locally $C^{1}$ and the surface is globally $G^{1}$. Before expanding

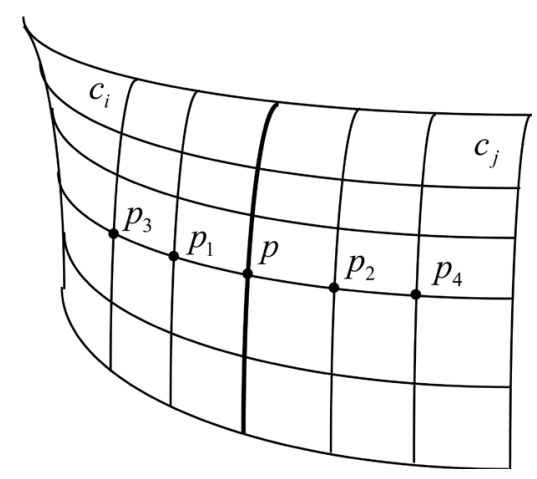

Figure 1. Schematic representation of the neighborhood of the assessed point $p$, a common point of two adjoining patches, $c_{i}$ and $c_{j}$. The part left to the thick curve belongs to $c_{i}$, and the part right to the thick curve belongs to $c_{j}$. Points $p_{1}$ and $p_{2}$ are at the first column in the neighborhood of the assessed point $p$ while points $p_{3}$ and $p_{4}$ are at the second column. 
Remark 1 into the bi-parametric curve segments, let us see the bi-parametric curves in a different way.

There exists a patch $c_{j} \subset \hat{S}$, where $c_{j}$ is locally $C^{1}$ and $\hat{S}$ is globally $G^{1}$. In the patch $c_{j}$, let us take a bi-parametric curve segment, $l_{c j}(w, t) \subset c_{j}$, in the way of Remark 2.

Remark 2. If we choose two different natural ( $\operatorname{arc})$ parameters along two different directions as two parameters of $w$ and $t$, respectively, two families of curves are corresponding to the network of two coordinates, $w$ and $t$.

Let $\varepsilon_{w}$ and $\varepsilon_{t}$ be assigned values in two sets of graduations, $E_{w}$ and $E_{t}$, respectively. That is, $\varepsilon_{w} \in E_{w}$ and $\varepsilon_{t} \in E_{t}$. If $\varepsilon_{w}$ is given a definite value in the set $E_{w}, l_{c j}\left(w=\varepsilon_{w}, t\right)$ represent a family of curves, that is, a family of $t$ coordinate segments. If $\varepsilon_{t}$ is given a definite value in the set $E_{t}, l_{c j}\left(w, t=\varepsilon_{t}\right)$ represent a family of $w$ coordinate segments. A coordinate network of $t$ and $w$ is built up with $l_{c j}\left(w=\varepsilon_{w}, t\right)$ and $l_{c j}\left(w, t=\varepsilon_{t}\right)$, which is shown in Figure 1. In the coordinate network, any one of these segments, $l_{c j . t}\left(w=\varepsilon_{w}, t\right)$ and $l_{c j . w}\left(w, t=\varepsilon_{t}\right)$, can be seen as a uni-parametric curve and Remark 1 can be applied to them.

Since each of parameters, $w$ and $t$, is a natural (arc) parameter,

$$
\begin{gathered}
l_{c j}\left(w=\varepsilon_{w}, t+\delta_{t}\right)-\left.l_{c j}\left(w=\varepsilon_{w}, t\right) \rightarrow \mathrm{d} l_{c j . t}\right|_{\left(\varepsilon_{w}, t\right)} \\
\text { and }\left|\left(\left.\mathrm{d} l_{c j . t}\right|_{\left(\varepsilon_{w}, t\right)}\right)\right| \rightarrow|\mathrm{d} t| \text { if } \delta_{t} \rightarrow 0 .
\end{gathered}
$$

and

$$
\begin{gathered}
l_{c j}\left(w+\delta_{w}, t=\varepsilon_{t}\right)-\left.l_{c j}\left(w, t=\varepsilon_{t}\right) \rightarrow \mathrm{d} l_{c j . w}\right|_{\left(w, \varepsilon_{t}\right)} \\
\quad \text { and }\left|\left(\left.\mathrm{d} l_{c j . w}\right|_{\left(w, \varepsilon_{t}\right)}\right)\right| \rightarrow|\mathrm{d} w| \text { if } \delta_{w} \rightarrow 0 .
\end{gathered}
$$

As $l_{c j}$ is expressed as radius vector,

$$
\left.\frac{\mathrm{d}}{\mathrm{d} t} l_{c j . t}\right|_{\left(\varepsilon_{w}, t\right)}=\left.\frac{\partial}{\partial t} l_{c j}\right|_{\left(\varepsilon_{w}, t\right)} \text {, and }\left.\frac{\mathrm{d}}{\mathrm{d} w} l_{c j . w}\right|_{\left(w, \varepsilon_{t}\right)}=\left.\frac{\partial}{\partial w} l_{c j}\right|_{\left(w, \varepsilon_{t}\right)},
$$

where $\left.\frac{\partial}{\partial t} l_{c j}\right|_{\left(\varepsilon_{w}, t\right)}$ and $\left.\frac{\partial}{\partial w} l_{c j}\right|_{\left(w, \varepsilon_{t}\right)}$ are the corresponding partial derivative vectors of the radius vector $l_{c j}$.

If the $\hat{S}$ is globally $C^{1}$, with Equation (1), we can find a bi-parametric curve, $l_{c i}(u, v) \subset c_{i} \subset \hat{S}$, which meets $l_{c j}(w, t) \subset c_{j} \subset \hat{S}$ at the common point $p=l_{c i}(\widehat{u}, \widehat{v})=l_{c j}(\widehat{w}, \widehat{t})$, and satisfies the following identities,

$$
\begin{aligned}
& \left.\frac{\partial}{\partial u} l_{c i}\right|_{p}=\left.\frac{\partial}{\partial w} l_{c j}\right|_{p}, \\
& \text { and }\left.\frac{\partial}{\partial v} l_{c i}\right|_{p}=\left.\frac{\partial}{\partial t} l_{c j}\right|_{p} .
\end{aligned}
$$

We should notice that $c_{i}$ also has the coordinate network of two corresponding coordinates, $u$ and $v$, and $\left.\frac{\partial}{\partial v} l_{c i}\right|_{\left(\varepsilon_{u}, v\right)}$ and $\left.\frac{\partial}{\partial u} l_{c i}\right|_{\left(u, \varepsilon_{v}\right)}$ are the corres- 
ponding partial derivative vectors.

In other words, the coordinate network of $c_{j}$ can be extended to its neighboring patches. That is, for any of $l_{c j . t}$ and $l_{c j . w}$, there exists a corresponding coordinate segment in its neighboring patches. Any of these coordinate segments can be the image of a segment $f$ defined in Definition 1 , which is continuous.

As a result of the above deduction, the $\hat{S}$ of the globally $C^{1}$ appears to be a globally smooth surface rather than a composite surface with the visibly changing when crossing the boundaries of patches. Neighboring patches of $\hat{S}$ have the consistently changing in the geometric sense.

Now let us back to Remark 1 and expand it into bi-parametric curve segments in two adjoining patches of a surface.

Remark 3. The idea of Remark 1 can be expanded into bi-parametric curve segments in two adjoining patches of a composite surface with two conditions and an adjusted result. The two conditions are that the patches are locally $C^{1}$ and the composite surface is globally $G^{1}$. The adjusted result is that the coordinate derivative vectors can twist and/or scale when crossing the boundary between a pair of adjoining patches. That is Proposition 1 as follows.

Proposition 1. If patches of a composite surface $\hat{S}$ are locally $C^{1}$ and $\hat{S}$ is globally $G^{1}$, the coordinate derivative vectors of bi-parametric curve segments in adjoining patches can twist andlor scale when crossing the boundary between a pair of adjoining patches.

The simplified proof is as follows. The idea will be extended gradually in the deduction of Remark 4 and Remark 5.

Proof. There exist a pair of adjoining patches $\left\{c_{i}, c_{j}\right\}, c_{i} \subset \hat{S}$ and $c_{j} \subset \hat{S}$. In these two patches, let us take two bi-parametric curve segments, $l_{c i}(u, v) \subset c_{i}$ and $l_{c j}(w, t) \subset c_{j}$ such that they meet at a common point $p=l_{c i}(\widehat{u}, \widehat{v})=l_{c j}(\widehat{w}, \widehat{t})$ and satisfy the $G^{1}$ condition, Equation (6), at the point $p$. In the differential geometric convention and theory of manifolds, $l_{c i}$ or $l_{c j}$ can be represented by the radius vector of any point along them, respectively.

Since patches are locally $C^{1}$, we can choose $u$ and $v$ to be two natural parameters along two different arc orientations in $c_{i}$, and choose $w$ and $t$ to be two natural parameters along two different arc orientations of $c_{j}$.

In the analysis of Remark 2, we know that in $c_{j}$ the coordinate network of $t$ and $w$ is composed of $l_{c j}\left(w=\varepsilon_{w}, t\right)$ and $l_{c j}\left(w, t=\varepsilon_{t}\right)$. In $c_{i}$, the coordinate network of $v$ and $u$ is made up of $l_{c i}\left(u=\varepsilon_{u}, v\right)$ and $l_{c i}\left(u, v=\varepsilon_{v}\right)$, where $\varepsilon_{u}$ and $\varepsilon_{v}$ be assigned values in two sets of graduations, $E_{u}$ and $E_{v}$, respectively. The corresponding partial derivative vectors are as follows,

$$
\left.\frac{\partial}{\partial t} l_{c j}\right|_{\left(\varepsilon_{w}, t\right)} \text { and }\left.\frac{\partial}{\partial w} l_{c j}\right|_{\left(w, \varepsilon_{t}\right)} \text { of } l_{c j} \text {, and }\left.\frac{\partial}{\partial v} l_{c i}\right|_{\left(\varepsilon_{u}, v\right)} \text { and }\left.\frac{\partial}{\partial u} l_{c i}\right|_{\left(u, \varepsilon_{v}\right)} \text { of } l_{c i} \text {. }
$$

Since $l_{c i}$ and $l_{c j}$ meet at the common point $p=l_{c i}(\hat{u}, \widehat{v})=l_{c j}(\widehat{w}, \hat{t})$ and $\hat{S}$ is globally $G^{1}, l_{c i}$ and $l_{c j}$ are two adjoining segments of one curve $l_{c i-j} \subset \hat{S}$, which is $G^{1}$. 
Equation (6) is satisfied at the common point $p$ when crossing two patches, $c_{i}$ and $c_{j}$. Let us individually rewrite each of two formulae in Equation (6) as follows,

$$
\begin{array}{r}
\left.\frac{\partial}{\partial u} l_{c i}\right|_{p}=\left.\left(\frac{\partial w}{\partial u} \cdot \frac{\partial}{\partial w} l_{c j}+\frac{\partial t}{\partial u} \cdot \frac{\partial}{\partial t} l_{c j}\right)\right|_{p}, \\
\text { and }\left.\frac{\partial}{\partial v} l_{c i}\right|_{p}=\left.\left(\frac{\partial w}{\partial v} \cdot \frac{\partial}{\partial w} l_{c j}+\frac{\partial t}{\partial v} \cdot \frac{\partial}{\partial t} l_{c j}\right)\right|_{p} .
\end{array}
$$

Let us examine the geometric meanings of Equations (6), (10) and (11). $\frac{\partial l_{c j}}{\partial w}, \frac{\partial l_{c j}}{\partial t}, \frac{\partial l_{c i}}{\partial u}$ and $\frac{\partial l_{c i}}{\partial v}$ are partial derivative vectors and have their own directions in $R^{3} . \frac{\partial w}{\partial u}, \frac{\partial t}{\partial u}, \frac{\partial w}{\partial v}$ and $\frac{\partial t}{\partial v}$ are parameters that can be changed with varied sizes of patches. Equation (10) represents that the vector $\frac{\partial l_{c i}}{\partial u}$ is the linear combination of two vectors, $\frac{\partial l_{c j}}{\partial w}$ and $\frac{\partial l_{c j}}{\partial t}$, rather than the identity of $\frac{\partial l_{c j}}{\partial w}$ in Equation (8), at the common point $p$. Similarly, Equation (11) represents that $\frac{\partial l_{c i}}{\partial v}$ is the linear combination of $\frac{\partial l_{c j}}{\partial w}$ and $\frac{\partial l_{c j}}{\partial t}$, rather than the identity of $\frac{\partial l_{c j}}{\partial t}$ in Equation (9), at the common point $p$.

In other words, with globally $G^{1}$, the pair of partial derivative vectors of each coordinate network hold the linear relationships with the corresponding partial derivative vectors of its neighboring patches.

Let us see carefully the matrix $T_{11}$ of Equation (6). With the theories of modern geometry and transformation group [9], if the square matrix $T_{11}$ is full rank, $T_{11}$ can perform twisting or scaling on a vector by left multiplying the vector.

Therefore, unlike a surface of the globally $C^{1}$, when a coordinate curve segment in a patch of $\hat{S}$ is extended to the corresponding coordinate curve segment in its neighboring patch, it may twist or scale.

These observations are just what is said in Proposition 1. That is, parameters, $\left.\frac{\partial w}{\partial u}\right|_{p},\left.\frac{\partial w}{\partial v}\right|_{p},\left.\frac{\partial t}{\partial u}\right|_{p}$, and $\left.\frac{\partial t}{\partial v}\right|_{p}$, help expand the identity of partial derivative vectors of the same orientation and scale under the globally $C^{1}$ condition into the equivalence of partial derivative vectors with twisting and/or scaling under the globally $G^{1}$ condition in three-dimensional space $R^{3}$. The latter situation results in the fact that the corresponding partial derivative vectors may twist or scale when crossing the boundary between a pair of neighboring patches.

Therefore, Proposition 1 has been proved.

Now, let us take the natural parameter $x$ as the arc of $l_{c j}$ in $c_{j}$ at the point $p$ and consider the coordinate network mentioned in Remark 2. Since $c_{j}$ is locally $C^{1}$, we can write the following formula, 


$$
\frac{\mathrm{d} l_{c j}}{\mathrm{~d} x}=\frac{\partial l_{c j}}{\partial w} \cdot \frac{\mathrm{d} w}{\mathrm{~d} x}+\frac{\partial \mathrm{l}_{c j}}{\partial t} \cdot \frac{\mathrm{d} t}{\mathrm{~d} x}
$$

From Equation (12) and $\frac{\mathrm{d} l_{c j}}{\mathrm{~d} x}$ is a unity vector, the square of the differential arc length can be written as

$$
\begin{aligned}
\mathrm{d} x^{2} & =\mathrm{d} l_{c j}^{2}=\left(\frac{\partial l_{c j}}{\partial w} \mathrm{~d} w+\frac{\partial l_{c j}}{\partial t} \mathrm{~d} t\right) \cdot\left(\frac{\partial l_{c j}}{\partial w} \mathrm{~d} w+\frac{\partial l_{c j}}{\partial t} \mathrm{~d} t\right) \\
& =\left(\frac{\partial l_{c j}}{\partial w} \bullet \frac{\partial l_{c j}}{\partial w}\right) \mathrm{d} w^{2}+2 \cdot\left(\frac{\partial l_{c j}}{\partial w} \bullet \frac{\partial l_{c j}}{\partial t}\right) \mathrm{d} w \mathrm{~d} t+\left(\frac{\partial l_{c j}}{\partial t} \bullet \frac{\partial l_{c j}}{\partial t}\right) \mathrm{d} t^{2}
\end{aligned}
$$

where $l_{c j}$ is the radius vector and $\left(\frac{\partial l_{c j}}{\partial w} \bullet \frac{\partial l_{c j}}{\partial t}\right)$ is the dot product of two partial derivative vectors, $\frac{\partial l_{c j}}{\partial w}$ and $\frac{\partial l_{c j}}{\partial t}$. Equation (13) is also known as the first fundamental form.

Remark 4. With the theory of the first fundamental form of a surface, a surface with the globally $C^{1}$ continuity holds the differential arc length. That is, both the orientation and size of differential arc are constant. In other words, if we expect to join two patches together with the differential arc length being invariant, these two patches must keep the orientation and size of the differential arc constant when crossing the boundary between them. From the analysis of Remark 3 and Equation (13), $\left.\mathrm{d} l_{c i}^{2}\right|_{p}=\left.\mathrm{d} l_{c j}^{2}\right|_{p}$ for joining $c_{i}$ and $c_{j}$ with the $C^{1}$ continuity.

This case is also called distance-preserving. The differential arc lengths, angles and areas are invariant when connecting patches together to construct a composite surface if these patches keep the first fundamental forms invariant. The composite surface is $C^{1}$.

What about only holding the orientation of the differential arc when crossing the boundary between two adjoining patches? The answer to this question is in the following Remark 5 .

Remark 5. If only the differential arc orientation is held, one of two adjoining patches, $c_{i}$ and $c_{j}$, has to be changed its own dimensions by being mapped or transformed into the other's. Or, they keep the difference of their dimensions and each of them holds its own distinct geometric details. With the theory of differential geometry [10], the condition of holding only differential arc orientation also means that

$$
\mathrm{d} w: \mathrm{d} t=\mathrm{d} u: \mathrm{d} v
$$

at the point $p=l_{c i}(\widehat{u}, \widehat{v})=l_{c j}(\widehat{w}, \widehat{t})$. In this situation, we will give the Proposition 2 and prove that this condition is equivalent to two conditions of Remark 3 , i.e. locally $C^{1}$ and globally $G^{1}$.

This case is also referred to as angle-preserving.

Proposition 2. The condition of angle-preserving is equivalent to the conditions that patches of a composite surface $\hat{S}$ are locally $C^{1}$ and the surface $\hat{S}$ 
is globally $G^{1}$.

The angle-preserving case may not be as obvious as the distance-preserving one in Remark 4. The simplified proof is given as follows.

Proof. From the definition of angle-preserving in the theory of differential geometry, it results in the fact that the sufficient and necessary conditions of angle-preserving are to hold the same ratio of the first fundamental forms. That is, given two first fundamental forms of the two joined patches, $c_{i}$ and $c_{j}$, which have been defined in Equation (13), they can be written as follows,

$$
\begin{aligned}
I_{c i} & =\mathrm{d} l_{c i}^{2}=E_{c i} \mathrm{~d} u^{2}+2 F_{c i} \mathrm{~d} u \mathrm{~d} v+G_{c i} \mathrm{~d} v^{2}, \\
\text { and } I_{c j} & =\mathrm{d} l_{c j}^{2}=E_{c j} \mathrm{~d} w^{2}+2 F_{c j} \mathrm{~d} w \mathrm{~d} t+G_{c j} \mathrm{~d} t^{2}
\end{aligned}
$$

where $\quad E_{c i}=\frac{\partial l_{c i}}{\partial u} \bullet \frac{\partial l_{c i}}{\partial u}, \quad F_{c i}=\frac{\partial l_{c i}}{\partial u} \bullet \frac{\partial l_{c i}}{\partial v}, \quad G_{c i}=\frac{\partial l_{c i}}{\partial v} \bullet \frac{\partial l_{c i}}{\partial v}, \quad E_{c j}=\frac{\partial l_{c j}}{\partial w} \bullet \frac{\partial l_{c j}}{\partial w}$, $F_{c j}=\frac{\partial l_{c j}}{\partial w} \bullet \frac{\partial l_{c j}}{\partial t}$, and $G_{c j}=\frac{\partial l_{c j}}{\partial t} \bullet \frac{\partial l_{c j}}{\partial t}$. And $I_{c i} / I_{c j}=\mathrm{d} l_{c i}^{2} / \mathrm{d} c_{c j}^{2}=c$ at the common point $p$, where $c$ is a constant. With Equation (6), the $G^{1}$ condition, replace $u$ and $v$ with $w$ and $t$, respectively, in Equation (15). And using Equation (14), the following equation can be led.

$$
\left.\frac{E_{c i}}{E_{c j}}\right|_{p}=\left.\frac{F_{c i}}{F_{c j}}\right|_{p}=\left.\frac{G_{c i}}{G_{c j}}\right|_{p} .
$$

Equation (17) is just the sufficient and necessary conditions for angle-preserving.

We have discussed the case that the patches of a composite surface are locally $C^{1}$ and the composite surface is globally $G^{1}$. Now let us take that idea a step further and think about the situations of $C^{2}$ and $G^{2}$ in the next section.

\section{Properties of Second-Order Continuities}

According to the theory of differential geometry [10] [11], if $\hat{S}$ is globally $C^{2}$, the second fundamental form of any curve $l_{c j} \subset c_{j} \subset \hat{S}$ is written as follows,

$$
I I_{c j}=L_{c j} \mathrm{~d} w^{2}+2 M_{c j} \mathrm{~d} w \mathrm{~d} t+N_{c j} \mathrm{~d} t^{2},
$$

where $L_{c j}=\frac{\partial^{2} l_{c j}}{\partial w^{2}} \bullet \boldsymbol{n}, M_{c j}=\frac{\partial^{2} l_{c j}}{\partial w \partial t} \bullet \boldsymbol{n}, N_{c j}=\frac{\partial^{2} l_{c j}}{\partial t^{2}} \bullet \boldsymbol{n}$, and $\boldsymbol{n}$ is the unit normal vector at the point $p=l_{c j}(\widehat{w}, \widehat{t})$. The geometric meaning of the second fundamental form is the degree of a point deviating from the tangent plant of $\hat{S}$ at the point $p$ when it is flowing along $l_{c j}$. We call it the bending rate in this paper.

Remark 6. With the theory of the second fundamental forms of a surface, a surface with the globally $C^{2}$ holds the bending rate of any arc at any given point of $\hat{S}$. Here, the bending rate of an arc represents the degree of the arc bending. In other words, the arc's changing rate keeps invariant when a point moving along any curve from a given point of $\hat{S}$. 
When considering the curve $l_{c i-j}$ formed by connecting $l_{c i}$ and $l_{c j}$ together at the common point $p=l_{c i}(\widehat{u}, \widehat{v})=l_{c j}(\widehat{w}, \widehat{t})$ and two adjoining patches $c_{i}$ and $c_{j}$, where $l_{c i} \subset c_{i}$ and $l_{c j} \subset c_{j}$, we can represent Remark 6 as follows,

$$
\left.I I_{c i}\right|_{p}=\left.I I_{c j}\right|_{p} \text {. }
$$

In Equation (19), $\left.I I_{c i}\right|_{p}$ is the second fundamental form at the point of $p=l_{c i}(\widehat{u}, \widehat{v})$ on the side of $c_{i}$, which is $I I_{c i}=L_{c i} \mathrm{~d} u^{2}+2 M_{c i} \mathrm{~d} u \mathrm{~d} v+N_{c i} \mathrm{~d} v^{2}$, where $L_{c i}=\frac{\partial^{2} l_{c i}}{\partial u^{2}} \bullet \boldsymbol{n}, M_{c i}=\frac{\partial^{2} l_{c i}}{\partial u \partial v} \bullet \boldsymbol{n}$ and $N_{c i}=\frac{\partial^{2} l_{c i}}{\partial v^{2}} \bullet \boldsymbol{n} .\left.\quad I I_{c j}\right|_{p}$ is the second fundamental form at the point of $p$ on the side of $c_{j}$, which is $I I_{c j}=L_{c j} \mathrm{~d} w^{2}+2 M_{c j} \mathrm{~d} w \mathrm{~d} t+N_{c j} \mathrm{~d} t^{2}$, where $L_{c j}=\frac{\partial^{2} l_{c j}}{\partial w^{2}} \bullet \boldsymbol{n}, M_{c j}=\frac{\partial^{2} l_{c j}}{\partial w \partial t} \bullet \boldsymbol{n}$, and $N_{c j}=\frac{\partial^{2} l_{c j}}{\partial t^{2}} \bullet \boldsymbol{n}$.

With the condition of Equation (19), both the orientation and size of the bending rate are held. In other words, if we expect to join two patches together with the bending rate invariant, these two patches must hold the orientation and size of the bending rate when crossing the boundary between them.

This case is called bending-rate-preserving in this paper. That is, the bending rate is kept when a point flowing crossing the boundary between two adjoining patches. In other words, the bending rate is invariant at a common point of two connected patches of a composite surface if the connected patches keep the second fundamental forms invariant at the common point. The composite surface is globally $C^{2}$.

It may be too stiff for researchers to construct a composite surface to hold the globally $C^{2}$ continuity.

Alternatively, what about only holding the bending orientation of the natural arc when crossing the boundary between two adjoining patches? The answer to this question is in the following Remark 7. We will check a composite surface that is locally $C^{2}$ and globally $G^{2}$.

Remark 7. If only the bending orientation is kept, one of two patches, $c_{i}$ and $c_{j}$, has to change the dimensions of its bending rate by mapping or transforming it into the other's. Or, they keep the difference of these dimensions and each of them holds its own changing rate.

In this paper, this case is called normal-section-curvature-preserving (in short NSC-preserving). Later in this section, we will give the reason for naming this term in this way. Let us study in details the condition of the NSC-preserving.

Proposition 3. Let $c_{i}$ and $c_{j}$ be a pair of adjoining patches of the composite surface $\hat{S}$. The condition of which $\hat{S}$ is NSC-preserving is equivalent to the conditions that patches of the composite surface $\hat{S}$ are locally $C^{2}$ and the composite surface $\hat{S}$ is globally $G^{2}$. That is, Equations (5), (6), and (7) are satisfied for any pair of $c_{i}$ and $c_{j}$ in $\hat{S}$.

Proof. It does not lose the generality to form $l_{c i-j}$ by connecting two seg- 
ments $l_{c i}$ and $l_{c j}$ that can map into a straight line in the tangent plane of $\hat{S}$ at the point $p=l_{c i}(\widehat{u}, \widehat{v})=l_{c j}(\widehat{w}, \hat{t})$. With this aim, we can try to find $l_{c i-j}$ by choosing $l_{c i} \subset c_{i}$ and $l_{c j} \subset c_{j}$ in such a way that each of them can map onto a segment of the same line of the tangent plane on one side of the point $p$. This type of segments exist on both sides of the boundary between $c_{i}$ and $c_{j}$ since the locally $C^{2}$ of $C_{i}$ and $C_{j}$, and globally $G^{2}$ of $\hat{S}$.

With the theory of the first and second fundamental forms of a surface, the condition that an adjoining pair of $c_{i}$ and $c_{j}$ of $\hat{S}$ is NSC-preserving can be written as follows,

$$
\left.\frac{I I_{c i}}{I_{c i}}\right|_{p}=\left.\frac{I I_{c j}}{I_{c j}}\right|_{p}
$$

where $\left.\frac{I I_{c i}}{I_{c i}}\right|_{p}=\left.\frac{L_{c i} \mathrm{~d} u^{2}+2 M_{c i} \mathrm{~d} u \mathrm{~d} v+N_{c i} \mathrm{~d} v^{2}}{E_{c i} \mathrm{~d} u^{2}+2 F_{c i} \mathrm{~d} u \mathrm{~d} v+G_{c i} \mathrm{~d} v^{2}}\right|_{p}$, and

$$
\left.\frac{I I_{c j}}{I_{c j}}\right|_{p}=\left.\frac{L_{c j} \mathrm{~d} w^{2}+2 M_{c j} \mathrm{~d} w \mathrm{~d} t+N_{c j} \mathrm{~d} t^{2}}{E_{c j} \mathrm{~d} w^{2}+2 F_{c j} \mathrm{~d} w \mathrm{~d} t+G_{c j} \mathrm{~d} t^{2}}\right|_{p}
$$

In the proof of the angle-preserving in Remark 4 of Section 6, we have proved that the globally $G^{1}$ continuity has the identities, $\left.\frac{\mathrm{d} u}{\mathrm{~d} v}\right|_{p}=\left.\frac{\mathrm{d} w}{\mathrm{~d} t}\right|_{p}$ and $\left.\frac{E_{c i}}{E_{c j}}\right|_{p}=\left.\frac{F_{c i}}{F_{c j}}\right|_{p}=\left.\frac{G_{c i}}{G_{c j}}\right|_{p}$ (Equations (14) and (17)). Therefore, it is required here only to prove the next identities,

$$
\left.\frac{L_{c i}}{L_{c j}}\right|_{p}=\left.\frac{M_{c i}}{M_{c j}}\right|_{p}=\left.\frac{N_{c i}}{N_{c j}}\right|_{p} .
$$

Equation (21) can be rewritten as follows,

$$
\frac{\left(\frac{\partial^{2} l_{c i}}{\partial u^{2}} \bullet \boldsymbol{n}\right)}{\left(\frac{\partial^{2} l_{c j}}{\partial w^{2}} \bullet \boldsymbol{n}\right)}=\left.\frac{\left(\frac{\partial^{2} l_{c i}}{\partial u \partial v} \bullet \boldsymbol{n}\right)}{\left(\frac{\partial^{2} l_{c j}}{\partial w \partial t} \bullet \boldsymbol{n}\right)}\right|_{p}=\left.\frac{\left(\frac{\partial^{2} l_{c i}}{\partial v^{2}} \bullet \boldsymbol{n}\right)}{\left(\frac{\partial^{2} l_{c j}}{\partial t^{2}} \bullet \boldsymbol{n}\right)}\right|_{p} .
$$

We right-dot-multiply each formula of matrix Equation (7) by $\boldsymbol{n}$. Note that the normal vector $\boldsymbol{n}$ is perpendicular to any tangent vector at the point $p$ and their dot products are zero. Thus, we can get the following formulae,

$$
\left\{\begin{array}{l}
\left.L_{c i}\right|_{p}=\left.\left[\left(\frac{\partial w}{\partial u}\right)^{2} L_{c j}+2\left(\frac{\partial w}{\partial u} \cdot \frac{\partial t}{\partial u}\right) M_{c j}+\left(\frac{\partial t}{\partial u}\right)^{2} N_{c j}\right]\right|_{p} \\
\left.M_{c i}\right|_{p}=\left.\left[\left(\frac{\partial w}{\partial u} \cdot \frac{\partial w}{\partial v}\right) L_{c j}+\left(\frac{\partial w}{\partial u} \cdot \frac{\partial t}{\partial v}+\frac{\partial w}{\partial v} \cdot \frac{\partial t}{\partial u}\right) M_{c j}+\left(\frac{\partial t}{\partial u} \cdot \frac{\partial t}{\partial v}\right) N_{c j}\right]\right|_{p} \\
\left.N_{c i}\right|_{p}=\left.\left[\left(\frac{\partial w}{\partial v}\right)^{2} L_{c j}+2\left(\frac{\partial w}{\partial v} \cdot \frac{\partial t}{\partial v}\right) M_{c j}+\left(\frac{\partial t}{\partial v}\right)^{2} N_{c j}\right]\right|_{p}
\end{array}\right.
$$


By considering $\mathrm{d} u: \mathrm{d} v=\mathrm{d} w: \mathrm{d} t$ at the common point $p$, Equations (21) or (22) can be got.

Thus, the proof is finished.

With the first and second fundamental forms, another useful geometrical property of $\hat{S}$ can be written as follows,

$$
k_{c j} \cos \theta_{c j}=I I_{c j} / I_{c j}
$$

where $k_{c j}$ is the curvature of the curve $l_{c j}$ at the point $p$, and $\theta_{c j}$ is the included angle between $\boldsymbol{n}$ and the principal normal vector $\beta_{c j}$ of the curve $l_{c j}$ at the point $p$.

Let $\bar{P}$ be the tangent plane of $\hat{S}$ at the point $p$. Let $\hat{l}_{c i-j}$ be the normal section that is tangent to $l_{c i-j}$ at the point $p$ and $k_{n}$ be the curvature of $\hat{l}_{c i-j}$. With Equations (20) and (24), we can have the following equations,

$$
k_{n}=k_{c i} \cos \theta_{c i}=k_{c j} \cos \theta_{c j},
$$

where $k_{c i}\left(\right.$ or $k_{c j}$ ) is the curvature of $l_{c i}\left(\right.$ or $\left.l_{c j}\right)$, and $\theta_{c i}\left(\right.$ or $\left.\theta_{c j}\right)$ is the angle between the normal vector $\boldsymbol{n}$ and $\beta_{c i}$ (or $\beta_{c j}$ ). $\beta_{c i}$ (or $\beta_{c j}$ ) is principal normal vector of $l_{c i}$ (or $l_{c j}$ ) at the point $p$, respectively.

Equation (25) gives the reason why we have the term, NSC-preserving (normal-section-curvature-preserving) for the case that the bending orientation is kept.

In the next section, we will give some examples of composite surfaces with definite continuities.

\section{Application Examples of Surface Continuities}

We use three examples to show how to apply the theory in Sections 6 and 7.

Example 1. The study of Lávička et al. [12] presents a scheme of smooth surface interpolation using patches with rational offsets. Their scheme is the Hermit interpolation with polynomial Pythagorean normal vectors. The surfaces constructed with their method have rational offsets at given points in a rectangular grid, which can give local geometrical details. Their method can produce local patches with piecewise rational $C^{1}$ and the composite surface with global $G^{1}$. Their method is a simple functional algorithm for computing composite surfaces without the need for any subsequent re-parameterizing or trimming of the parameter domain. In their paper [12], we can see that patches with Pythagorean-normal-vector method interpolating the same boundary data may bring out a smooth composite surface by a suitable choice of the tangent vectors or produce a ridge look with the specific choice (shown in Figure 8 in their paper [12]). For the situation of locally $C^{1}$ in patches and globally $G^{1}$ in the composite surface, Remark 3 and Remark 5 have given a detailed explanation.

Example 2. In the study of Shen et al. [13], they address a method that converts multiple NURBS (non-uniform rational B-splines) patches to a single untrimmed NURBS-compatible subdivision surface in order to avoid a gap between a pair of adjacent NURBS patches, which are usually trimmed and stitched 
by the standard CAD tools. In their method, they adopt quadrangle patches and bivariate parameterization (i.e. bi-parametric).

First of all, a gap between a pair of adjacent NURBS patches is resulted from breaking the $C^{0}$ continuity condition when crossing the boundary between these two patches. In other words, the NURBS patches are trimmed without globally holding the $C^{0}$ continuity.

In the procedure of Shen et al. [13], a coarse quadrilateral mesh is created first from the trimmed bi-cubic NURBS patches. Then, this coarse mesh is refined to form a non-uniform subdivision mesh that is maintained to retain the boundary geometry property of each NURBS patch through boundary curve spacing and surface fitting. In mathematics sense, the method maps a collection of trimmed bi-cubic NURBS patches into a single subdivision surface of the non-uniform subdivision mesh.

A single subdivision surface with the global $C^{1}$ can be produced by merging the subdivision patches (see Figure 12 in their study [13]). Even better, some of their converted model can maintain inter-patch continuity up to the $C^{2}$ continuity (see Figures 1 and 17 in their study [13]).

As mentioned in their paper, in some cases, pre-processing and inter-fitting are necessary to satisfy the smoothness requirements. That is, they pre-process the coarse mesh of NURBS and trim it into the subdivision mesh; and then fit the subdivision surface with tight tolerance and fairness functions to approximate the collection of NURBS patches. The former brings out a new knot mesh that replaces the original NURBS one. The latter produces a new subdivision surface that substitutes the collection of NURBS patches to represent a target surface in a visibly smooth way.

The authors indicated that since the process of trimming for $C^{1}$ and $C^{2}$ might change the model edge curve, they regarded the maintenance of smoothness as more important than exactly fitting the edge curve, and their models of $C^{1}$ (see Figure 12 in their study [13]) and $C^{2}$ (see Figures 1 and 17 in their study [13]) were obtained in this way.

Example 3. The study of Liu [14] presented the Progressive and Mixing Algorithm (PAMA) which can give the inter-patch $C^{0}$ continuity for a ridge looking, or the inter-patch $G^{1}$ continuity for a smooth look in different cases. PAMA adopts bi-parameterization and quadrangle patches. With the Betasplines [3] [4] [15] which have applied geometric continuities to curve and surface construction, PAMA was created for surface construction and edition with human-computer interaction. Without the cost of trimming, PAMA has been applied to the real-time FPGA (field programmable gate array) implementation.

\section{Results and Conclusions}

In this paper, we have explored two classes of continuities, parametric continuities and geometric continuities less than and equal to second order, and their geometric properties. The presented propositions can be applied to verify the 
continuities of surfaces constructed in computer. We can summarize the geometric properties presented in this paper as follows.

$C^{0}$ and $G^{0}$ continuities can be satisfied by the connection of two adjoining patches with a sharp look such as a ridge, along their common boundary.

$C^{1}$ continuity can be satisfied by identifying the first parametric derivatives on both sides along the common boundary, which restricts the parametric representations of the first column points on both sides around a boundary point of the adjoining patches. The first column points have been shown in Figure 1 in Section 5.

$G^{1}$ continuity can be satisfied by aligning the first parametric derivatives on both sides along the boundary with the flexibility given by four arguments of the first inter-parametric partial derivatives (see Equation (6)), which relaxes the restriction carried by $C^{1}$ continuity but maintains appropriately a smooth look similar to $C^{1}$ continuity.

$C^{2}$ continuity can be achieved by identifying the second parametric partial derivatives on both sides along the common boundary, which heavily restricts the parametric representations of the first two columns of points on both sides around a boundary point of the adjoining patches. This stiff condition can be met by constructing a surface as a whole set of bi-parametric cubic representations. The first and second column points are shown in Figure 1 in Section 5.

$G^{2}$ continuity can be attained by aligning the first and second parametric partial derivatives on both sides along the common boundary with the flexibility given by two matrices of parameters involved with the first and second inter-parametric partial derivatives, that is, parameters of the parametric partial derivatives (see Equation (7)), which relaxes the stiff restriction of $C^{2}$ continuity but holds a smooth look similar to $C^{2}$ continuity.

The study in this paper is limited to the geometric properties of continuities less than and equal to second order. The properties of continuities of higher order have not been found in this paper.

In future, we will keep doing research on bridging the gap between the existent mathematics theories and the surface construction in computer, and extend the study on a composite surface into its algebraic topology. Its purpose is to find a mathematical basis to make a comparison between a composite surface and its target surface that would be represented by the composite surface.

\section{Acknowledgements}

This work has been supported by Hebei Project of Sponsoring High-Level Talents [grant number C2015003045], Doctoral Research Foundation of Hebei University of Science and Technology [grant number 201417], and Hebei Project of Sponsoring Introduced Overseas Researchers [grant number C201811].

\section{Conflicts of Interest}

The authors declare no conflicts of interest regarding the publication of this paper. 


\section{References}

[1] Schmidt, P., Born, J., Campen, M. and Kobbelt, L. (2019) Distortion-Minimizing Injective Maps between Surfaces. ACM Transactions on Graphics (TOG), 38, Article No. 156. https://doi.org/10.1145/3355089.3356519

[2] Karčiauskas, K. and Peters, J. (2019) Curvature-Bounded Guided Subdivision: Biquartics vs Bicubice. Computer-Aided Design, 114, 122-132.

https://doi.org/10.1016/j.cad.2019.05.011

[3] Barsky, B.A. and DeRose, T.D. (1989) Geometric Continuity of Parametric Curves: Three Equivalent Characterizations. IEEE Computer Graphics and Application, 9, 60-68. https://doi.org/10.1109/38.41470

[4] Barsky, B.A. and DeRose, T.D. (1990) Geometric Continuity of Parametric Curves: ConStructions of Geometrically Continuous Splines. IEEE Computer Graphics and Application, 10, 60-68. https://doi.org/10.1109/38.45811

[5] Farin, G. (1982) Visually C2 Cubic Splines. Computer-Aided Design, 14, 137-139. https://doi.org/10.1016/0010-4485(82)90326-8

[6] Farin, G. (1993) Curves and Surfaces for Computer Aided Geometric Design, a Practical Guide. 3rd Edition, Academic Press Inc., New York. https://doi.org/10.1016/B978-0-12-249052-1.50005-2

[7] Hoschek, J. and Lasser, D. (1993) Fundamentals of Computer Aided Geometric Design. A K Peters Ltd., Wellesley.

[8] Massey, W.S. (2009) Algebraic Topology: An Introduction. Springer-Verlag, Berlin/Heidelberg/New York and World Publishing Company of Beijing, Beijing.

[9] Dubuluowen, B.A., Roweikefu, C.N. and Fumingke, A.T. (2006) Modern Geometry-Methods and Applications, Part I: The Geometry of Surfaces, Transformation Groups and Fields. High Education Press, Beijing.

[10] Spivak, M. (1999) A Comprehensive Introduction to Differential Geometry, Vol. 3. 3rd Edition, Publish or Perish, Wilmington.

[11] Verpoort, S. (2008) Geometry of the Second Fundamental Form: Curvature Properties and Variational Aspects. Doctoral Thesis, University of Leuven, Belgium.

[12] Lávička, M., Šír, Z. and Vršek, J. (2016) Smooth Surface Interpolation Using Patches with Rational Offset. Computer Aided Geometric Design, 48, 75-85. https://doi.org/10.1016/j.cagd.2016.09.002

[13] Shen, J., Kosinka, J., Sabin, M. and Dodgson, N. (2016) Converting a CAD Model into a Non-Uniform Subdivision Surface. Computer Aided Geometric Design, 48, 17-35. https://doi.org/10.1016/j.cagd.2016.07.003

[14] Liu, Y. (2013) A Novel Parallel Algorithm for Surface Editing and Its FPGA Implementation. Doctoral Thesis, University of Bedfordshire, Luton. http://ethos.bl.uk/OrderDetails.do?uin=uk.bl.ethos.606677

[15] Joe, B. (1990) Knot Insertion for Beta-Spline Curves and Surfaces. ACM Transactions on Graphics ( TOG), 9, 41-45. https://doi.org/10.1145/77635.77638 